\title{
EFEITO DA LUZ E DA TEMPERATURA DE CONGELAMENTO SOBRE A ESTABILIDADE DAS ANTOCIANINAS DA PITANGA ROXA ${ }^{1}$
}

\author{
Vera Lúcia Arroxelas G. de LIMA ${ }^{2, *}$, Enayde de Almeida MÉLO², Daisyvângela E. da Silva LIMA²
}

\section{RESUMO}

As antocianinas são corantes naturais que podem ser utilizadas como alternativa em substituição aos corantes sintéticos. Entretanto, a baixa estabilidade destes compostos é o principal fator que dificulta sua aplicação como corante em alimentos. Frente à escassez de estudo sobre o pigmentos antociânicos da pitanga roxa, este trabalho teve como propósito avaliar a sua estabilidade à luz e ao congelamento. Utilizando a leitura de absorbância do extrato antociânico submetido às condições experimentais foi evidenciado que a luz reduziu o seu tempo de meia-vida, enquanto que a temperatura de congelamento exerceu pequeno efeito na degradação dos pigmentos.

Palavras-chave: Eugenia uniflora L.; estabilidade à luz; estabilidade ao congelamento.

\section{SUMMARY}

THE EFFECTS OF LIGHT AND FREEZING TEMPERATURE UPON THE STABILITY OF PURPLE SURINAM CHERRY'S ANTHOCYANINS. Anthocyanins are natural colorants that can be used as alternative to synthetic dyes. However, the low stability of these compounds is the main drawback in their application as food colorants. Since the information on anthocyanin pigments in Surinam cherry is scarce, the purpose of this investigation was to assess their stability to light and freezing storage. The anthocyanin extract absorbance, under the experimental conditions, showed that light incidence reduced the half-life of anthocyanin extract; meanwhile the freezing storage had little effect on these pigments degradation.

Keywords: Eugenia uniflora L.; light stability; frozen stability.

\section{1 - INTRODUÇÃO}

Frente a atual tendência mundial em usar pigmentos naturais como corantes para alimentos, ênfase tem sido dado a busca de fontes economicamente viáveis. Neste contexto, destacam-se as antocianinas, compostos fenólicos derivados do cátion favilium, pertencentes ao grupo dos flavonóides, largamente distribuídas na natureza, que conferem a muitos vegetais as várias nuanças de cores entre laranja, vermelho e azul [8]. O interesse por estes pigmentos decorre também de evidências relacionadas ao seu potencial benéfico à saúde em virtude de sua ação antioxidante [7, 21].

O uso de extratos antociânicos como corantes naturais é limitado em decorrência da grande instabilidade desses compostos a vários fatores, dentre os quais destacam-se a luz e a temperatura de congelamento. A instabilidade à luz do extrato antociânico purificado do açaí foi constatado por BOBBIO et al. [3]. SILVA, MENEZES \& GUEDES [18] fazem referência a redução do teor de antocianinas em polpa de acerola, congelada por 6 meses.

A pitangueira, fruteira nativa das regiões Sul e Sudeste do Brasil, cujos frutos são bastante apreciados devido ao seu sabor doce e ácido agradável e aroma peculiar intenso, tem se adaptado favoravelmente às condições climáticas e edáficas da região Nordeste. Durante o processo de maturação da pitanga, a cor do epicarpo evolui

\footnotetext{
1. Recebido para publicação em 27/05/2003. Aceito para publicação em 22/12/2004 (001139).

2. DCD - Universidade Federal Rural de Pernambuco (UFRPE). Rua Dom Manoel de Medeiros s/n, Dois Irmãos, CEP: 52171-900, Recife-PE, Brasil, E-mail: veraarroxelas@hotmail.com

* A quem a correspondência deve ser enviada.
}

de verde a vermelha, podendo chegar até quase negra [2]. No Brasil não se conhecem variedades perfeitamente definidas de pitangueira e em Pernambuco, é comum encontrar fruto de coloração variando de alaranjada a avermelhada [12]. No entanto, estes autores relatam a existência de pitanga de cor roxa, pigmentação decorrente da presença de antocianinas [14]. Frente à escassez de estudos sobre o pigmento antociânico da pitanga roxa, este trabalho teve como objetivo avaliar a sua estabilidade à luz e à temperatura de congelamento.

\section{2 - MATERIAL E MÉTODOS}

\section{1 - Preparo das amostras}

Pitangas roxas, no estádio maduro, procedentes de um pomar localizado em Paratibe-PE, foram colhidas no período da manhã. Os frutos $(2 \mathrm{Kg})$ foram transformados em polpa utilizando uma centrifuga doméstica. Parte desta polpa, acondicionada em potes plásticos $(30 \mathrm{~g})$, foi armazenada sob congelamento $\left(-18^{\circ} \mathrm{C}\right)$, em "freezer". A outra parte, foi utilizada para obtenção do extrato antociânico bruto que foi submetido ao ensaio de sua estabilidade à luz.

\section{2- Estabilidade do pigmento antociânico ao con- gelamento}

Unidades amostrais da polpa congelada ( 3 potes) foram retiradas ao acaso, no tempo zero e após 2, 4, 6 e 12 meses de estocagem. Para quantificação de antocianinas totais, o extrato antociânico bruto foi obtido homogeneizando a polpa em solução extratora (etanol 95\%: $\mathrm{HCl} 1,5 \mathrm{~N})(85: 15 \mathrm{v} / \mathrm{v})$, que foi deixado em repouso por $12 \mathrm{~h} \mathrm{a} 5^{\circ} \mathrm{C}$ ao abrigo da luz. Em seguida, foi filtrado e o resíduo exaustivamente lavado com a solução ex- 
tratora até a remoção completa do pigmento. O extrato bruto foi submetido à leitura espectrofotométrica a $535 \mathrm{~nm}$ de acordo com método descrito por LEES \& FRANCIS [13] e o teor de antocianinas totais quantificado utilizando a seguinte expressão:

$$
\begin{aligned}
& \text { Antocianinas totais }=\text { Absorbância } X \text { fator de diluiçăo } \\
& \text { (mg. } 100 \mathrm{~g}^{-1} \text { ) } \quad \mathrm{E}^{1 \%}{ }_{1 \mathrm{~cm}}
\end{aligned}
$$

O valor $\mathrm{E}^{1 \%}{ }_{1 \mathrm{~cm}}$ utilizado foi de 98,2 , conforme estabelecido por LEES \& FRANCIS [13].

As determinações foram efetuadas em triplicata e os dados obtidos, submetidos à Análise de Variância e as médias comparadas pelo Teste de Tukey ao nível de $5 \%$ de probabilidade, utilizando o programa estatístico "minitab-10 for Windows".

\section{3- Estabilidade do pigmento antociânico à luz}

O extrato antociânico bruto $(\mathrm{pH} 1,0)$, obtido conforme explicitado no item anterior, foi distribuído em 12 tubos de ensaio $(20 \mathrm{~mL})$ com tampas de borracha e mantidos a temperatura ambiente de $27 \pm 1^{\circ} \mathrm{C}$. Seis tubos foram expostos à luz fluorescente (duas lâmpadas paralelas de $80 \mathrm{~W}$ ) que se encontrava à distância de $1,34 \mathrm{~m}$, e os outros seis tubos foram mantidos ao abrigo da luz, cobertos cuidadosamente com tecido "black out" (100\% polipropileno). Periodicamente, a leitura da absorbância dos extratos foi realizada a $535 \mathrm{~nm}$ e os dados utilizados para o cálculo da constante de velocidade $(\mathrm{k})$ e do tempo de meia-vida $\left(\mathrm{t}_{1 / 2}\right)$ dos extratos através de equações cinéticas de $1^{\mathrm{a}}$ ordem [16]:

$$
\begin{aligned}
& -\ln \left[\frac{\text { Absorbância }(\mathrm{t})}{\text { Absorbância }\left(\mathrm{t}_{0}\right)}\right]=\mathrm{k} \mathrm{t} \\
& \mathbf{t}_{1 / 2}=\frac{\ln 2}{\mathrm{k}}
\end{aligned}
$$$$
\text { (Equação 1) }
$$$$
\text { (Equação 2) }
$$

\section{3 - RESULTADOS E DISCUSSÃO}

Na polpa de pitanga armazenada sob congelamento ocorreu degradação de antocianinas (Figura 1), de modo significativo aos 60 dias de estocagem, entretanto o teor do pigmento manteve-se praticamente constante $(p>0,05)$ nos meses subseqüentes. Embora a degradação inicial tenha sido estatisticamente significativa, com percentual de perda de $8,77 \%$, a coloração do produto não apresentou alterações visualmente perceptiveis. Outras frutas armazenadas sob congelamento, em forma de polpa, suco ou in natura, a perda do teor antociânico foi superior a evidenciada neste estudo. O percentual de redução deste constituinte para o suco de "cranberry" foi de 40\%, para cereja ácida da cultivar "Érdi" correspondeu a $58 \%$ e para polpa de acerola foi de $21,74 \%$ após congelamento por 1, 4 e 6 meses, respectivamente [18, 19, 20].

LIMA, MÉLO \& LIMA [15] detectaram na pitanga roxa um teor de fenólicos totais de $325 \mathrm{mg} / 100 \mathrm{~g}$. A estabilidade do pigmento antociânico deste fruto durante o congelamento pode ter sido decorrente da presença destes compostos, tendo em vista que os mesmos podem atuar como co-pigmentos. DAVIES \& MAZZA [5], referem que a complexação molecular de antocianinas com outros fenólicos é o principal mecanismo de estabilização da cor. Segundo MAZZA \& BROUILLARD [17], o pH é o fator que mais afeta a cor das antocianinas em solução que em meio ácido apresenta a cor vermelha, tornando-se azulada em $\mathrm{pH}$ neutro e, em alcalino, incolor. O pH 3,1 da polpa da pitanga roxa [14], considerado ácido, também favoreceu a estabilidade da cor da polpa deste fruto submetida ao congelamento.

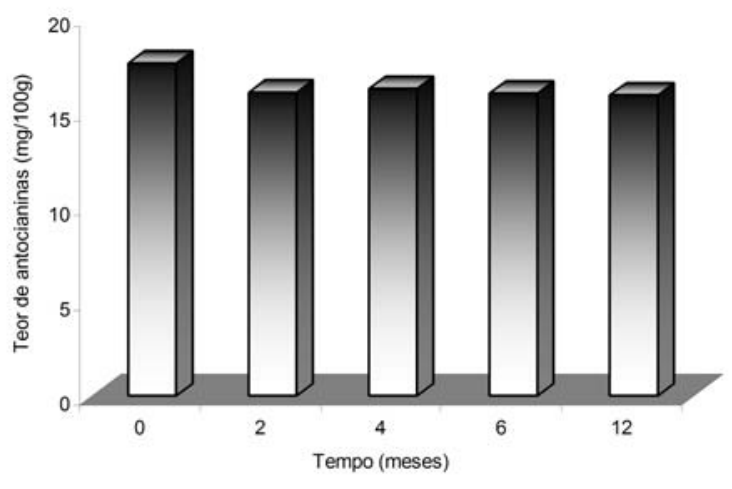

FIGURA 1. Teor de antocianinas totais da polpa de pitanga roxa submetida ao congelamento $\left(-18^{\circ} \mathrm{C}\right)$ por 12 meses

A estrutura quimica das antocianinas da pitanga roxa pode ser outro fator que contribuiu para a sua maior estabilidade. A presença de açúcares, de açúcares acilados, de grupos metoxil e hidroxil têm efeito marcante na cor e na reatividade das antocianinas [17]. Segundo FRANCIS [8], aquelas que apresentam maior número de hidroxilas são menos estáveis do que as que possuem maior número de metoxilas. O grau de glicosilação é outra característica estrutural que favorece a estabilidade dessas moléculas, sendo as diglicosiladas mais estáveis do que as monoglicosiladas. As antocianinas aciladas retêm melhor a cor, mesmo em $\mathrm{pH}$ alcalino ou sob ação de outros fatores como aquecimento, luz e $\mathrm{SO}_{2}[6,8,10]$. INAMI et al. [9] constataram que antocianinas aciladas, em sistema modelo (bebida), apresentaram maior estabilidade à luz e ao calor. Desta forma, possivelmente as antocianinas desse fruto encontram-se aciladas e apresentam grupos metoxil em suas moléculas, o que será confirmado posteriormente. Neste sentido, estudos adicionais estão sendo desenvolvidos neste laboratório para caracterizar as antocianinas da pitanga roxa.

O extrato antociânico de pitanga roxa ao abrigo da luz manteve-se mais estável do que o extrato submetido à luminosidade (Tabela 1). As constantes de velocidade $(\mathrm{k})$ foram $7,3 \times 10^{-4}$ e $5,7 \times 10^{-4} \mathrm{~h}^{-1}$ para o primeiro e segundo extrato, respectivamente. A perda de $50 \%$ da cor original (tempo de meia) ocorreria, portanto, após 948,3h (39 dias) para o extrato submetido ao efeito da luz e após 1205,7h (50 dias) para o extrato ao abrigo da luz, demonstrando a maior instabilidade do extrato antociânico à luminosidade. 
TABELA 1. Teor de antocianinas totais e perda de absorbância do extrato de pitanga roxa submetida à presença e ausência de luz

\begin{tabular}{|c|c|c|c|c|c|c|}
\hline \multirow{2}{*}{$\begin{array}{r}\text { Tempo } \\
\text { (horas) }\end{array}$} & \multicolumn{3}{|c|}{ Presença de luz } & \multicolumn{3}{|c|}{ Ausência de luz } \\
\hline & $\begin{array}{c}\text { Absorbância } \\
(535 \mathrm{~nm})\end{array}$ & $\begin{array}{c}\text { Antocianinas } \\
\left(\mathrm{mg} \mathrm{l}^{2} 00 \mathrm{~g}^{-1}\right)\end{array}$ & $\begin{array}{c}\text { Perda } \\
(\%)\end{array}$ & $\begin{array}{c}\text { Absorbância } \\
(535 \mathrm{~nm})\end{array}$ & $\begin{array}{c}\text { Antocianinas } \\
\left(\mathrm{mg} 100 \mathrm{~g}^{-1}\right)\end{array}$ & $\begin{array}{c}\text { Perda } \\
(\%)\end{array}$ \\
\hline 0 & 0,765 & 16,23 & - & 0,765 & 16,23 & - \\
\hline 24 & 0,727 & 15,42 & 4,97 & 0,732 & 15,53 & 4,31 \\
\hline 98 & 0,662 & 14,05 & 13,46 & 0,683 & 14,49 & 10,72 \\
\hline 240 & 0,589 & 12,50 & 23,01 & 0,632 & 13,41 & 17,39 \\
\hline 432 & 0,525 & 11,14 & 31,37 & 0,598 & 12,69 & 21,83 \\
\hline 576 & 0,497 & 10,54 & 35,03 & 0,403 & 10,67 & 34,25 \\
\hline 720 & 0,461 & 9,78 & 39,74 & 0,479 & 10,16 & 37,39 \\
\hline 840 & 0,414 & 8,78 & 45,88 & 0,472 & 10,01 & 38,30 \\
\hline
\end{tabular}

O tempo de meia-vida para o extrato exposto à luz neste estudo foi superior ao do jambolão (430,09h), baguaçu $(508,06 \mathrm{~h})$ e uva $(207,98 \mathrm{~h})$ determinado por KUSKOSKI, MARQUES \& FETT. [11]. Estes autores, ao submeter o extrato de uva, sob atmosfera de nitrogênio, à ausência de luz, evidenciaram que o tempo de meia vida foi de 1290,50h. Este valor foi semelhante ao obtido para o da pitanga roxa em presença de oxigênio, o que permite inferir que este extrato poderia apresentar melhor estabilidade do que o de uva se o experimento tivesse sido conduzido nas mesmas condições.

BAILONI, BOBBIO \& BOBBIO [1] evidenciaram que os pigmentos antociânicos das folhas da Acalipha híspida, sob atmosfera de $\mathrm{N}_{2}$, atingiriam o tempo de meio vida a $2800 \mathrm{~h}$, superior portanto ao da pitanga roxa, no entanto este último encontrava-se em presença de oxigênio. O extrato antociânico bruto da vinagreira (Hibiscus sabdariffa L.) apresentou tempo de meia vida de $353 \mathrm{~h}$ em presença de luz/oxigênio e $\mathrm{pH} 2,3$, o qual foi reduzido a $81 \mathrm{~h}$ ao elevar o $\mathrm{pH}$ a 4,0 [4]. Evidencia-se, portanto que a estabilidade deste pigmento foi bastante influenciada pelo $\mathrm{pH}$.

$\mathrm{O}$ extrato antociânico de pitanga roxa surge como perspectiva para uso como corante natural em produtos alimentícios, acondicionados em embalagem opaca. Entretanto, outras condições experimentais devem ser avaliadas para o uso deste extrato em alimentos, tendo em vista que outros fatores, tais como $\mathrm{pH}$, temperatura, ausência de oxigênio, a forma em que deve ser adicionado ao produto, se como extrato ou microencapsulado, podem influenciar na estabilidade do pigmento.

\section{4 - CONCLUSÕES}

De acordo com os resultados obtidos pode-se concluir que a presença de luz reduziu o tempo de meia vida do extrato antociânico bruto da pitanga roxa. A temperatura de congelamento exerceu pequeno efeito sobre a degradação dos pigmentos antociânicos deste fruto.

\section{5 - REFERÊNCIAS BIBLIOGRÁFICAS}

[1] BAILONI, M. A.; BOBBIO, P. A.; BOBBIO, F. Preparação e estabilidade do extrato antociânico das folhas da Acalipha hispida. Ciênc. Tecnol. Aliment., v. 8, n. 1, p. $17-18,1998$.

[2] BEZERRA, J. E. F.; SILVA, J. R. J. F.; LEDERMAN, I. E. Pitanga (Eugenia uniflora L.). (Série Frutas Nativas, 1). Jaboticabal: Funep, 2000. 30p.
[3] BOBBIO, F. O.; FADELLI, S.; OLIVEIRA, P. A.; BOBBIO, P. A. Estudo da estabilidade das antocianinas da polpa de açaí (Euterpe oleracea). Anais do III Simpósio Latino Americano de Ciência de Alimentos, p. 55. 1999.

[4] CAMAREZ, R. R. B.; KUSKOSKI, E. M.; BRIGHENTE, I. M. C.; FETT, R. Estabilidade das antocianinas do cálice de Hibiscus sabdariffa L. Rev. Bras. Cor. Nat., v. 4, n. $1 / 2$, p. 67-72, 2000.

[5] DAVIS, A. J.; MAZZA, G. Copigmentation of simple and acylated anthocyanins with colorless phenolic compounds. J. Agric. Food Chem., v. 41, n. 5, p. 716-720, 1993.

[6] DELGADO-VARGAS, F.; JIMÉNEZ, A. R.; PAREDESLÓPEZ, O. Natural pigments: carotenoids, anthocyanins, and betalains- characteristics, biosynthesis, processing, and storage. Crit. Rev. Food Sci. Nutr., v. 40, n. 3, p. 173-289, 2000.

[7] ESPÍN, J. C.; SOLER-RIVAS, C.; WICHERS, H. J.; GARCÍA-VIGUERA, C. Anthocyanin-based natural colorants: a new source of antiradical activity for foodstuff. J. Agric. Food Chem., v. 48, n. 5, p. 15881592, 2000.

[8] FRANCIS, F. J. Food colorants: anthocyanins. Crit. Rev. Food Sci. Nutr., v. 28, n. 4, p. 273-314, 1989.

[9] INAMI, O.; TAMURA, I.; KIKUZAKI, H.; NAKATANI, N. Stability of anthocyanins of Sambucus canadensis and Sambucus nigra. J. Agric. Food Chem., v. 44, n. 10, p. 3090-3096, 1996.

[10] JACKMAN, R. L.; YADA, R.Y.; TUNG, M. A.; SPEERS, R. A. Anthocyanins as food colorants- A review. J. Food Biochem., v. 11, p. 201-247, 1987.

[11] KUSKOSKI, E. M.; MARQUES, P. T.; FETT, R. Estudo comparativo da estabilidade das antocianinas do baguaçu, jambolão e da uva. Rev. Bras. Cor. Nat., v. 4. n. $1 / 2$, p. $73-76,2000$.

[12] LEDERMAN, I. E.; BEZERRA, J. E. F.; CALADO, G. A pitangueira em Pernambuco. Recife: Secretaria de Agricultura, 1992. 20p. Documentos, 19.

[13] LEES, D. H.; FRANCIS, F. J. Standardization of pigment analyses in cranberries. HortScience, v. 7 , n. 1, p. 83-84, 1972.

[14] LIMA, V. L. A. G.; MÉLO, E. A.; LIMA, L. S.; NASCIMENTO, P. P. Caracterização físico-química e sensorial de pitanga roxa. Rev. Bras. Frutic., v. 22, n. 3, p. 382-385, 2000.

[15] LIMA, V. L. A. G.; MÉLO, E. A.; LIMA, D. E. S. Fenólicos e carotenóides totais em pitanga. Sci. Agric., v. 59, n. 3, p. 447-450, 2002.

[16] MAHAN, B. M.; MYERS, R. J. Cinética química. In: MAHAN, B. M.; MYERS, R. J. (Ed). Química: um curso universitário. São Paulo: Edgard Blücher Ltda, 1993. p 233-265.

[17] MAZZA, G.; BROUILLARD, R. Recent developments in the stabilization of anthocyanins in food products. Food Chem., v. 25, p. 207-225, 1987.

[18] SILVA, M. F. V.; MENEZES, H. C.; GuEDES, M. C. Efeito de diferentes tratamentos térmicos sobre as antocianinas na polpa de acerola. Anais do III Simpósio Latino Americano de Ciência de Alimentos, p. 152-153, 1999.

[19] STARR, M. S.; FRANCIS, F. J. Oxygen and ascorbic acid effect on the relative stability of four anthocyanin pigments in cranberry juice. Food Technol., v. 22, p. 91-93, 1968.

[20] URBÁNYI, G.; HORTI, K. Changes of surface colour of the fruit and of the anthocyanin content of sour cherries during frozen storage. Acta Alimentaria, v. 21, n. 34, p. 307-323, 1992.

[21] WANG, H.; CAO, G.; PRIOR, R. L. Total antioxidant capacity of fruits. J. Agric. Food Chem., v. 44, n. 3, p. 701-705, 1996. 